\title{
Band Distributions for Quantum Chaos on the Torus
}

\author{
Itzhack Dana, ${ }^{1}$ Mario Feingold, ${ }^{2}$ and Michael Wilkinson ${ }^{3}$ \\ ${ }^{1}$ Department of Physics, Bar-Ilan University, Ramat-Gan 52900, Israel \\ ${ }^{2}$ Department of Physics, Ben-Gurion University, Beer-Sheva 84105, Israel \\ ${ }^{3}$ Department of Physics and Applied Physics, University of Strathclyde, Glasgow G4 ONG, U. K.
}

\begin{abstract}
Band distributions (BDs) are introduced describing quantization in a toral phase space. $\mathrm{A} \mathrm{BD}$ is the uniform average of an eigenstate phase-space probability distribution over a band of toral boundary conditions. A general explicit expression for the Wigner BD is obtained. It is shown that the Wigner functions for all of the band eigenstates can be reproduced from the Wigner BD. Also, BDs are shown to be closer to classical distributions than eigenstate distributions. Generalized BDs, associated with sets of adjacent bands, are used to extend in a natural way the Chern-index characterization of the classical-quantum correspondence on the torus to arbitrary rational values of the scaled Planck constant.
\end{abstract}

PACS numbers: 05.45.+b, 02.40.-k, 03.65.Ca, 03.65.Sq 
The classical and quantum dynamics of several nonintegrable model systems, which have become paradigmatic in the field of Quantum Chaos, can be reduced to a torus, either in configuration space (e.g., the Sinai billiard [1] 3]) or in phase space (e.g., the "cat maps" [4.5] and the "kicked-Harper" model [6 12]). Quantally, the admissible toral states have to satisfy proper boundary conditions (BCs), i.e., they have to be periodic in the torus up to constant Bloch phase factors. This Letter is concerned with systems for which general Bloch BCs are defined on a toral phase space: several of such systems have been discussed in the Quantum-Chaos literature [4-12], although attention has often been confined to strict periodicity. General Bloch BCs are physically relevant if the toral phase space represents the unit cell of a Hamiltonian or mapping which is periodic on phase space: models of this type occur naturally in problems involving magnetic fields combined with periodic potentials 13322 . A further reason for studying Bloch BCs is that some physical insight comes from examining the sensitivity of eigenstates to variation in the BCs [9 12,21].

It is well known [4 12] that a necessary condition for the reduction of phase-space quantum dynamics to a torus $T_{Q}^{2}$ is that a scaled Planck constant for the problem, denoted by $\rho=\hbar / 2 \pi$, assumes rational values: $\rho=q / p$ ( $q$ and $p$ are coprime integers). The "quantum" torus $T_{Q}^{2}$ is $q$ times larger than the torus $T^{2}$ to which the corresponding classical dynamics can be reduced [6, 10 12]. For each BC, characterized by the Bloch wavevector w, the spectrum consists of precisely $p$ levels [11]. As $\mathbf{w}$ is varied, these levels broaden into $p$ bands labeled by an index $b$. A measure of the sensitivity of the eigenstates in band $b$ to variations in the BCs is the Chern index $\sigma_{b}$ [9 12,21], an integer topological invariant analogous to the quantum Hall conductance carried by a magnetic band in a perfect crystal [13 20]. The eigenstates may be weakly dependent on the BCs only if $\sigma_{b}=0$, a value which may arise only if $q=1$ [11]. In this case, where $T_{Q}^{2}=T^{2}$, one can easily establish a classical-quantum correspondence on the torus for small $\rho$ [4,9:10]. Several arguments [9, 10,21], supported by numerical evidence, indicate that if the Husimi distribution of an eigenstate is localized on a classical regular orbit [e.g., a Kol'mogorov-Arnol'd-Moser torus or a periodic orbit] the 
corresponding band has $\sigma_{b}=0$. On the other hand, eigenstates whose Husimi distribution is spread over the classical chaotic region should belong to bands with $\sigma_{b} \neq 0$. The transition from a nearly-integrable regime, where almost all $\sigma_{b}=0$, to a fully chaotic regime, where almost all $\sigma_{b} \neq 0$, as a nonintegrability parameter is increased, takes place via degeneracies between adjacent bands, leading to a "diffusion" of the Chern indices [23]. The last three sentences summarize what we call the Chern-index characterization of the classical-quantum correspondence on the torus.

There is a sense in which eigenstates are not natural for a characterization of the classicalquantum correspondence, in that they exhibit rather nonclassical features, due to their association with the purely quantum quantity $\mathbf{w}$ and to their generic sensitivity on this quantity. In particular, the Chern-index characterization above cannot be extended to the general case $q \neq 1$ on the basis of the eigenstates [10,[1]. In order to take into account all the BCs but, at the same time, to eliminate their individual purely quantum effects, we propose in this Letter to characterize the classical-quantum correspondence on a toral phase space by quantities given by averages over all the BCs. As a matter of fact, one such quantity is the Chern index itself, which can be expressed as the uniform average of the eigenstate two-form with respect to $\mathbf{w}$ [9,11]. Here we introduce the band distribution (BD), given by the uniform average of the phase-space probability distributions (either Wigner or Husimi) for the band eigenstates over all the BCs. The BD may be viewed as the representative probability distribution for a level in the torus. We obtain a general explicit expression for the Wigner BD. A main result of this Letter is expressed by the formulae (3) and (6) below, by which the Wigner functions of all of the band eigenstates are encoded in a single, analytic function, the Wigner BD. Thus, no information is lost about the individual eigenstates by averaging the Wigner function over the band. Also, it will be demonstrated that the BDs are "more classical" than the distributions of band eigenstates in several aspects. We also give a generalization of the BD concept, which is analogous to the smoothing a probability distribution over a range of energy levels which is used in scar theory [24]. As a main application, the 
generalized BDs are used to extend in a very natural way the Chern-index characterization of the classical-quantum correspondence to $q \neq 1$. More details about the Husimi case are given in Ref. [12].

Our phase space is $(u, v),[\hat{u}, \hat{v}]=2 \pi \mathrm{i} \rho$, and the Hamiltonian or mapping for our system is assumed to be periodic in this space with a $2 \pi \times 2 \pi$ unit cell, which is the classical torus $T^{2}$. If $\rho=q / p$, the quantum torus can be chosen as $T_{Q}^{2}=[0,2 \pi q) \times[0,2 \pi)$ and the $v$-representation of the band eigenstates is [11]

$$
\Psi_{b, \mathbf{w}}(v)=\sum_{m=0}^{p-1} \phi_{b}(m ; \mathbf{w}) \sum_{l=-\infty}^{\infty} \exp \left[\mathrm{i} l\left(w_{1}+2 \pi m \rho\right) / q\right] \delta\left(v-w_{2}+2 \pi l / p\right),
$$

where $b=1, \ldots, p$. This state is an eigenfunction of the commuting phase-space translation operators on $T_{Q}^{2}, \hat{D}_{1}=\exp (\mathrm{i} \hat{u} / \rho)$ and $\hat{D}_{2}=\exp (\mathrm{i} p \hat{v})$, where $\hat{u}=2 \pi \mathrm{i} \rho d / d v$ : the eigenvalues are $\exp \left(\mathrm{i} w_{1} / \rho\right)$ and $\exp \left(\mathrm{i} p w_{2}\right)$, implying that $\mathbf{w}=\left(w_{1}, w_{2}\right)$ is the Bloch wavevector. Up to phase factors depending only on $\mathbf{w}$ and on the Chern index $\sigma_{b}$, the eigenstates (四) are periodic in w space with a unit cell given by the "Brillouin zone" $B Z=[0,2 \pi \rho) \times[0,2 \pi / p)$ [11. We denote by $P_{b, \mathbf{w}}(u, v)$ a phase-space probability distribution (either Wigner or Husimi) for the eigenstates. We define the band distribution (BD) for band $b$ by

$$
P_{b}(u, v)=\frac{1}{|B Z|} \int_{B Z} d \mathbf{w} P_{b, \mathbf{w}}(u, v)
$$

where $|B Z|=4 \pi^{2} q / p^{2}$ is the area of the Brillouin zone. A more explicit expression for $P_{b}(u, v)$ can be obtained in the Wigner case. We find in this case, using (11),

$$
\begin{aligned}
P_{b, \mathbf{w}}(u, v) \equiv & \frac{1}{2 \pi^{2} \rho} \int_{-\infty}^{\infty} d v^{\prime} \exp \left(\mathrm{i} u v^{\prime} / \pi \rho\right) \Psi_{b, \mathbf{w}}^{*}\left(v-v^{\prime}\right) \Psi_{b, \mathbf{w}}\left(v+v^{\prime}\right) \\
& =\sum_{r=-\infty}^{\infty} \sum_{s=-\infty}^{\infty} A_{b}(r, s ; \mathbf{w}) \delta\left(u-w_{1}-r \pi \rho\right) \delta\left(v-w_{2}-s \pi / p\right)
\end{aligned}
$$

where the first equality defines the Wigner function, and where

$$
A_{b}(r, s ; \mathbf{w})=\frac{p}{4 \pi} \sum_{m=0}^{p-1} \exp \left[\frac{\mathrm{i} \pi s(r-2 m)}{p}\right] \phi_{b}(m ; \mathbf{w}) \phi_{b}^{*}[(r-m) \bmod p ; \mathbf{w}] .
$$

The result (3) shows that the support of the Wigner function for an eigenstate is, quite generally, a lattice in phase space, which is shifted uniformly by shifting $\mathbf{w}$. This generalizes 
the result of Hannay and Berry 《4 for $\mathbf{w}=\mathbf{0}$ (strict periodicity) to arbitrary BCs. Using (3) in (2), we obtain the following expression for the Wigner BD:

$$
P_{b}(u, v)=\frac{p^{2}}{4 \pi^{2} q} \sum_{r=0,1} \sum_{s=0,1} A_{b}\left(r, s ; w_{1}=u-r \pi \rho, w_{2}=v-s \pi / p\right) .
$$

Equation (5) will now be inverted to express $A_{b}(r, s ; \mathbf{w})$ in terms of the Wigner BD. To this end, one notices first that, due to the strict periodicity of Eq. (3) in the Brillouin zone, the right-hand side of (国) will not change if the summations are performed over $r=\bar{r}, \bar{r}+1$ and $s=\bar{s}, \bar{s}+1$, where $\bar{r}$ and $\bar{s}$ are arbitrary integers. Next, one writes Eq. (5) with $(u, v)$ replaced by $(u+k q \pi, v+l \pi)$ for $k=0,1$ and $l=0,1$, choosing $\bar{r}=k p, \bar{s}=l p$ in the four cases. Using (耳), one then finds that $P_{b}(u+k q \pi, v+l \pi)$ is given by the right-hand side of Eq. (5) with the extra factor $(-1)^{k s+l r+k l p}$ under the summation signs. The resulting four equations can be easily solved for $A_{b}(r, s ; \mathbf{w})$ :

$$
A_{b}(r, s ; \mathbf{w})=\frac{\pi^{2} q}{p^{2}} \sum_{k=0,1} \sum_{l=0,1}(-1)^{k s+l r+k l p} P_{b}(u+k q \pi, v+l \pi),
$$

where $u=w_{1}+r \pi \rho$ and $v=w_{2}+s \pi / p$. Together with the latter expressions, Eq. (6) shows that the Wigner functions (3) of all the band eigenstates are simply encoded in the single, smooth phase-space function $P_{b}(u, v)$. Thus, no information about (3) is lost by performing the average in (2), as this information is fully recoverable from the Wigner BD.

We now discuss properties of the BD (目), including aspects in which it is "more classical" than $P_{b, \mathbf{w}}(u, v)$. First, we remark that $P_{b}(u, v)$, in particular (5), is a smooth function, unlike the Wigner function (3) of the eigenstates. Also, using the relation $e^{\mathrm{i} r\left(\hat{v}-w_{2}\right) / \rho} \Psi_{b, \mathbf{w}}(v)=\Psi_{b, w_{1}-2 \pi r, w_{2}}(v)$ ( $r$ integer) [11] in (2), and noticing that $\exp (\mathrm{i} r \hat{v} / \rho)$ is just a translation of $u$ by $2 \pi r$, we easily find that $P_{b}(u, v)$ is periodic with unit cell $T^{2}$ (the classical torus) for general $q$. This is in contrast with $P_{b, \mathbf{w}}(u, v)$, whose unit cell of periodicity is the quantum torus $T_{Q}^{2}$. We can then impose on $P_{b}(u, v)$ the normalization condition $\int_{T^{2}} d u d v P_{b}(u, v)=1$, making $P_{b}(u, v)$ analogous to a classical probability distribution in the phase space $T^{2}$. Using the periodicity of $P_{b}(u, v)$ in Eq. (6), it is easy to see that at 
any four points $(u+k q \pi, v+l \pi)(k=0,1, l=0,1)$ in $T_{Q}^{2}, P_{b, \mathbf{w}}(u, v)$ in (3) assumes values differing at most in sign, a fact which was observed in Ref. [4] in the particular case of $\mathbf{w}=\mathbf{0}$ (and $q=1$ ). This means that only a quarter of the values assumed by (3) in $T_{Q}^{2}$ may be independent. This rather nonclassical property of (3) is generally not possessed by the Wigner BD.

Next, consider $P_{b, \mathbf{w}}(u, v)$ in the Husimi case: $P_{b, \mathbf{w}}(u, v)=\left|\Psi_{b, \mathbf{w}}(u, v)\right|^{2}$, where $\Psi_{b, \mathbf{w}}(u, v)$ is the coherent-state representation of $\left|\Psi_{b, \mathbf{w}}\right\rangle$. For given $(b, \mathbf{w}), P_{b, \mathbf{w}}(u, v)$ always assumes $p$ zeros $\left(u_{0, j}, v_{0, j}\right)(j=1, \ldots, p)$ in $T_{Q}^{2}$ [9,11, 12]. These zeros make $P_{b, \mathbf{w}}(u, v)$ rather "nonclassical", for example, they do not allow $P_{b, \mathbf{w}}(u, v)$ to approach, in the semiclassical limit, the microcanonical uniform distribution in a strong-chaos regime [9,25]. On the other hand, the Husimi BD never vanishes $\left[P_{b}(u, v)>0\right.$ in $\left.T^{2}\right]$, simply because the $p$ zeros $\left(u_{0, j}, v_{0, j}\right)$ generally vary with $\mathbf{w}$ and the definition (2) involves an integration over all $\mathbf{w}$.

Finally, we discuss the important case of bands with Chern index $\sigma_{b}=0$, which is possible only when $q=1$ [1]. In this case, $\left|\Psi_{b, \mathbf{w}}\right\rangle$ can be written as a symmetry-adapted sum [16,18],

$$
\left|\Psi_{b, \mathbf{w}}\right\rangle=\sum_{l_{1}, l_{2}=-\infty}^{\infty} e^{-\mathrm{i} p\left(l_{1} w_{1}+l_{2} w_{2}\right)} \hat{D}_{1}^{l_{1}} \hat{D}_{2}^{l_{2}}\left|\varphi_{b}\right\rangle
$$

where $\left|\varphi_{b}\right\rangle$ is some square-integrable state, which is analogous to a Wannier function 16, 18, 20. Inserting (7) into (21), we easily obtain a general exact expression for the BD:

$$
P_{b}(u, v)=\sum_{l_{1}, l_{2}=-\infty}^{\infty} P_{\varphi_{b}}\left(u+2 \pi l_{1}, v+2 \pi l_{2}\right)
$$

where $P_{\varphi_{b}}(u, v)$ is the Wigner or Husimi function of the Wannier state $\left|\varphi_{b}\right\rangle$. While the Wannier function is not invariant under gauge transformations in which the Bloch states are multiplied by $\exp \left[\mathrm{i} \theta\left(w_{1}, w_{2}\right)\right]$ [20], the $\mathrm{BD}(\mathbb{8})$ is gauge invariant. In a nearly-integrable situation and in a semiclassical regime, the Husimi $P_{\varphi_{b}}(u, v)$ is well localized on a classical regular orbit, provided band $b$ is well separated from neighboring bands $\left[P_{\varphi_{b}}(u, v)\right.$ is then 
the "quasi-mode" of Ref. [26]]. In the semiclassical limit $\rho \rightarrow 0, P_{\varphi_{b}}(u, v)$ tends point-wise to zero outside the classical orbit [26]. Similarly, the BD (8) in the Husimi case tends pointwise to zero outside the periodic repetition of the classical orbit on all unit cells $\left(l_{1}, l_{2}\right)$. It is therefore a periodic version of the quasi-mode Husimi density, appropriate for a toral phase space. The difference $P_{b, \mathbf{w}}(u, v)-P_{b}(u, v)$ is the sum of the overlaps of the quasi-mode with the translated quasi-mode in all unit cells $\left(l_{1}, l_{2}\right) \neq(0,0)$, and it is of a purely quantum nature. This clarifies the classical nature of the BD in this case.

In some important cases, it is necessary to generalize the BD concept by averaging over more than one band, usually over a set of $N$ adjacent bands $b=b_{1}, \ldots, b_{N}$. This set may be considered as a single entity, a generalized band (GB), which can be characterized by its total Chern index, $\sigma_{\mathrm{GB}} \equiv \sum_{b=b_{1}}^{b_{N}} \sigma_{b}$, and by the generalized BD associated with it, $P_{\mathrm{GB}}(u, v)=N^{-1} \sum_{b=b_{1}}^{b_{N}} P_{b}(u, v)$. The further averaging over bands should give a "more classical" BD, as when smoothing over many levels in a general quantum system [24]. The "maximal" smoothing is, of course, that over all the $p$ bands. From the completeness of the eigenstates (四), we find in this case that $p^{-1} \sum_{b=1}^{p} P_{b}(u, v)=\left(4 \pi^{2}\right)^{-1}$. Thus, as one could expect, the generalized $\mathrm{BD}$ in this case is just the uniform distribution in phase space.

The use of generalized BDs is quite natural, for example, near a degeneracy between a pair of bands. In fact, precisely at the degeneracy point it is usually not useful to consider the two bands separately, and they must be treated as one single entity (the GB). It is well known [15] that the Chern indices of the two bands generically vary by \pm 1 (for $q=1$ ) across the degeneracy, leaving their total Chern index unchanged. Similarly, one can show [12] that the generalized BD for the two bands is approximately conserved across the degeneracy, despite the fact that the separate BDs may vary drastically.

We are now ready to present a main application of the BD concept. We show how generalized BDs can be used to extend in a natural way the Chern-index characterization 
of the classical-quantum correspondence on the torus [9 11] to general rational values $q^{\prime} / p^{\prime}$ of $\rho$ near the special values of the form $1 / p$ for which this characterization was originally formulated. Our basic assumption is that the renormalization-group approach developed in [19]20], which was applied to the investigation of the spectrum of a general class of Hamiltonians on the torus, is applicable to the band spectrum of our nonintegrable system. This assumption has been verified numerically for the kicked Harper model on a broad interval of the nonintegrability parameter [12,27]. Let $\rho^{\prime}=q^{\prime} / p^{\prime}$ be a rational number sufficiently close to $\rho=q / p$ and such that $p^{\prime} \gg p$. From Ref. [19], we know that the $p^{\prime}$ bands for $\rho^{\prime}$ can be grouped into $p$ "clusters" of adjacent bands, where each cluster $C_{b}$ is associated in a natural way with a band $b$ for $\rho=q / p$. Namely, the energy or quasienergy interval covered by the bands in $C_{b}$ is relatively close to that covered by band $b$ and the total Chern index $\sigma\left(C_{b}\right)$ of $C_{b}$ is equal to $\sigma_{b}$. The spectrum and eigenstates in $C_{b}$ can be calculated approximately from an effective Hamiltonian $H_{\text {eff }}$, obtained by properly quantizing the band function for band $b$.

The existence of $H_{\text {eff }}$ means that the space of states in band $b$ approximately coincides with the space of states in $C_{b}$. In other words, the projection operator for band $b$ is approximately equal to that for $C_{b}$ :

$$
\frac{1}{|B Z|} \int_{B Z} d \mathbf{w}\left|\Psi_{b, \mathbf{w}}\right\rangle\left\langle\Psi_{b, \mathbf{w}}\left|\approx \frac{1}{N_{b}} \sum_{b^{\prime}=d(b)}^{d(b)+N_{b}-1} \frac{1}{\left|B Z^{\prime}\right|} \int_{B Z^{\prime}} d \mathbf{w}^{\prime}\right| \Psi_{b^{\prime}, \mathbf{w}^{\prime}}^{\prime}\right\rangle\left\langle\Psi_{b^{\prime}, \mathbf{w}^{\prime}}^{\prime}\right|
$$

where all the primed quantities refer to $\rho^{\prime}, N_{b}$ is the number of bands in $C_{b}$, and $d(b)$ is the label of the lowest band in $C_{b}$. We immediately obtain from (9) that

$$
\frac{1}{|B Z|} \int_{B Z} d \mathbf{w}\left|\Psi_{b, \mathbf{w}}(u, v)\right|^{2} \approx \frac{1}{N_{b}} \sum_{b^{\prime}=d(b)}^{d(b)+N_{b}-1} \frac{1}{\left|B Z^{\prime}\right|} \int_{B Z^{\prime}} d \mathbf{w}^{\prime}\left|\Psi_{b^{\prime}, \mathbf{w}^{\prime}}^{\prime}\left(u^{\prime}, v^{\prime}\right)\right|^{2}
$$

where the variables $\left(u^{\prime}, v^{\prime}\right)$ for $\rho^{\prime}$ are related to the variables $(u, v)$ for $\rho$ by $\left(u^{\prime}, v^{\prime}\right)=$ $\sqrt{\rho / \rho^{\prime}}(u, v)$. Eq. (10) shows that the Husimi BD for band $b$ is approximately equal to the generalized Husimi $\mathrm{BD} P_{C_{b}}^{\prime}\left(u^{\prime}, v^{\prime}\right)$ for cluster $C_{b}$. An analogous approximate equality in the Wigner case can be similarly established. In the limit $\rho^{\prime} \rightarrow \rho$, the space of the clus- 
ter becomes identical to that of band $b$ [the approximate equality in (9) is replaced by an equality], and $P_{C_{b}}^{\prime}\left(u^{\prime}, v^{\prime}\right) \rightarrow P_{b}(u, v)$.

The most important reference values of $\rho$ are those with $q=1$, for which the Chern-index characterization of the classical-quantum correspondence is well established [9 [1]. For these $\rho$ 's, Eq. (10) implies that if the BD for band $b$ is concentrated, in a semiclassical regime, on a regular classical orbit $\left[\sigma_{b}=\sigma\left(C_{b}\right)=0\right]$ or on the classical chaotic region $\left[\sigma_{b}=\sigma\left(C_{b}\right) \neq 0\right]$, the same will be true for the generalized BD for $C_{b}$. One can also show that general cluster states, characterized by a well-defined value of w, are "weakly" or "strongly" sensitive to variations in $\mathbf{w}$ depending on whether $\sigma\left(C_{b}\right)=0$ or $\sigma\left(C_{b}\right) \neq 0$ [12]. The Chern-index characterization of the classical-quantum correspondence on the torus is thus extended to $\rho^{\prime}$ sufficiently close to $\rho=1 / p$ by replacing single bands $b$ with the corresponding clusters $C_{b}$.

In conclusion, the $\mathrm{BD}$ concept introduced in this Letter was shown to exhibit interesting and useful properties: (a) The Wigner functions of the band eigenstates are fully recoverable from the Wigner BD. (b) At the same time, the BDs are "more classical" than eigenstate distributions in several aspects. (c) Generalized BDs allow to extend in a natural way the Chern-index characterization of the classical-quantum correspondence to $q \neq 1$.

We remark that the BD concept could be further developed in several directions. Smoothing a quantum probability distribution over a range of energy levels is important in the theoretical study of scars using the semiclassical periodic-orbit theory [24]. In the case of the $\mathrm{BD}$, the smoothing is performed over the continuous range of one band, corresponding essentially to a single level in the framework of a toral phase space. This "minimal" smoothing is performed just for the sake to eliminate the purely quantum effects of individual BCs. Using the adaptation of periodic-orbit theory to the framework of a toral phase space [28], it may be possible to achieve a better understanding of the nature of BDs and generalized BDs in the semiclassical limit. Also, a more complete characterization of the classical-quantum 
correspondence on the phase-space torus should be achieved by introducing, in addition to the Chern index and the BDs, new quantities which are also naturally defined as averages over all the BCs. It should also be interesting to determine whether the distributions for the band eigenstates are also recoverable from the Husimi BD, and to extend the representation (8) of the $\mathrm{BD}$ in terms of Wannier states to the case $\sigma_{b} \neq 0$, using results from Ref. [20].

\section{Acknowledgments}

We would like to thank J. Zak, P. Leboeuf, A. Voros, M. V. Berry, and Y. Rutman for comments and discussions. ID and MF acknowledge support from the Israel Science

Foundation. MW acknowledges support from the EPSRC, grant GR/L/02302, and the hospitality of Ben-Gurion and Bar-Ilan Universities. 


\section{REFERENCES}

[1] Ya. G. Sinai, Russ Math. Surv. 25, 137 (1970).

[2] M. V. Berry, Ann. Phys. 131, 163 (1981).

[3] H. Primack and U. Smilansky, Phys. Rev. Lett. 74, 4831 (1995)

[4] J. H. Hannay and M. V. Berry, Physica (Amsterdam) 1D, 267 (1980).

[5] J. P. Keating, Nonlinearity 4, 309 (1991).

[6] R. Roncaglia, L. Bonci, F. M. Izrailev, B. J. West, and P. Grigolini, Phys. Rev. Lett. 73, $802(1994)$.

[7] I. Dana, Phys. Rev. Lett. 73, 1609 (1994).

[8] R. Artuso, G. Casati, F. Borgonovi, L. Rebuzzini, and I. Guarneri, Int. J. Mod. Phys. B 8, 207 (1994), and references therein.

[9] P. Leboeuf, J. Kurchan, M. Feingold, and D. P. Arovas, Phys. Rev. Lett. 65, 3076 (1990); ibid., Chaos 2, 125 (1992).

[10] F. Faure and P. Leboeuf, in From Classical to Quantum Chaos, Proceedings of the Conference, edited by G. F. Dell'Antonio, S. Fantoni, and V. R. Manfredi (SIF, Bologna, 1993), Vol. 41.

[11] I. Dana, Phys. Rev. E 52, 466 (1995).

[12] I. Dana, Y. Rutman, and M. Feingold, Phys. Rev. E 58, xxx (1998): chao-dyn/9810001 at http://xxx.lanl.gov/archive/chao-dyn/.

[13] D. J. Thouless, M. Kohmoto, M. P. Nightingale, and M. den Nijs, Phys. Rev. Lett. 49, 405 (1982).

[14] J. E. Avron, R. Seiler, and B. Simon, Phys. Rev. Lett. 51, 51 (1983).

[15] B. Simon, Phys. Rev. Lett. 51, 2167 (1983). 
[16] I. Dana and J. Zak, Phys. Rev. B 32, 3612 (1985), and references therein.

[17] I. Dana, Y. Avron, and J. Zak, J. Phys. C: Solid State Phys. 18, L679 (1985).

[18] M. Wilkinson, Proc. Roy. Soc. Lond. A403, 135 (1986).

[19] M. Wilkinson, J. Phys. A: Math. Gen. 20, 4337 (1987).

[20] M. Wilkinson, J. Phys. A: Math. Gen. 27, 8123 (1994).

[21] D. P. Arovas, R. N. Bhatt, F. D. M. Haldane, P. B. Littlewood, and R. Rammal, Phys. Rev. Lett. 60, 619 (1988).

[22] I. Dana, Phys. Lett. A 197, 413 (1995).

[23] P. N. Walker and M. Wilkinson, Phys. Rev. Lett. 74, 4055 (1995).

[24] M. V. Berry, Proc. Roy. Soc. London A 423, 219 (1989).

[25] P. Leboeuf and A. Voros, J. Phys. A: Math. Gen. 23, 1765 (1990).

[26] F. Faure, J. Phys. A: Math. Gen. 27, 7519 (1994).

[27] I. Dana (unpublished).

[28] P. Leboeuf and A. Mouchet, Phys. Rev. Lett. 73, 1360 (1994); G. Junker and H. Leschke, Physica (Amsterdam) 56D, 135 (1992). 